\title{
Experimental studies of Debye-like process and structural relaxation in mixtures of 2-ethyl-1-hexanol and 2-ethyl-1-hexyl bromide
}

\author{
M. Preuß, ${ }^{1}$ C. Gainaru, ${ }^{1, a)}$ T. Hecksher, ${ }^{2}$ S. Bauer, ${ }^{1}$ J. C. Dyre, ${ }^{2}$ R. Richert, ${ }^{3}$ \\ and R. Böhmer ${ }^{1}$ \\ ${ }^{1}$ Fakultät für Physik, Technische Universität Dortmund, 44227 Dortmund, Germany \\ ${ }^{2}$ DNRF Centre "Glass and Time”, IMFUFA, Department of Sciences, Roskilde University, P.O. Box 260, \\ DK-4000 Roskilde, Denmark \\ ${ }^{3}$ Department of Chemistry and Biochemistry, Arizona State University, Tempe, Arizona 85287-1604, USA
}

(Received 26 July 2012; accepted 13 September 2012; published online 9 October 2012)

\begin{abstract}
Binary solutions of 2-ethyl-1-hexanol $(2 \mathrm{E} 1 \mathrm{H})$ with 2-ethyl-1-hexyl bromide $(2 \mathrm{E} 1 \mathrm{Br})$ are investigated by means of dielectric, shear mechanical, near-infrared, and solvation spectroscopy as well as dielectrically monitored physical aging. For moderately diluted 2E1H the slow Debye-like process, which dominates the dielectric spectra of the neat monohydroxy alcohol, separates significantly from the $\alpha$-relaxation. For example, the separation in equimolar mixtures amounts to four decades in frequency. This situation of highly resolved processes allows one to demonstrate unambiguously that physical aging is governed by the $\alpha$-process, but even under these ideal conditions the Debye process remains undetectable in shear mechanical experiments. Furthermore, the solvation experiments show that under constant charge conditions the microscopic polarization fluctuations take place on the time scale of the structural process. The hydrogen-bond populations monitored via near-infrared spectroscopy indicate the presence of a critical alcohol concentration, $x_{c} \approx 0.5-0.6$, thereby confirming the dielectric data. In the pure bromide a slow dielectric process of reduced intensity is present in addition to the main relaxation. This is taken as a sign of intermolecular cooperativity probably mediated via halogen bonds. ( 2012 American Institute of Physics. [http://dx.doi.org/10.1063/1.4755754]
\end{abstract}

\section{INTRODUCTION}

For the structure and dynamics of bio-macromolecules, water, and many glass forming liquids hydrogen bonds play an important role. However, even focusing on the probably simpler case of small-molecule glass formers, such as the classical glass formers propylene glycol or glycerol that feature several hydroxyl groups per molecule, it is often found that they exhibit a more complex behavior than molecular liquids devoid of hydrogen bonds: The behavior of such hydrogen-bonded liquids often displays deviations from various scaling properties and they are thus considered peculiar viscous liquids. ${ }^{1}$ Moreover, substances with only a single hydroxyl group per molecule, such as the monohydroxy alcohols, reveal an even more interesting behavior. When monitoring their response using dielectric spectroscopy, not only the structural relaxation as well as faster modes can be detected but additionally an intense low-frequency absorption shows up. This relaxational feature, named after Peter Debye who formulated the theoretical description of its spectral shape almost 100 years ago, ${ }^{2}$ is known experimentally at least since the late 1920s. ${ }^{3}$ Despite considerable experimental ${ }^{4}$ and theoretical ${ }^{5,6}$ effort its microscopic nature had remained controversial for decades. Recently, with a renewed interest in these also technologically relevant solvents, monohydroxy alcohols have been explored using fresh approaches and wit-

\footnotetext{
a) Author to whom correspondence should be addressed. Electronic mail: catalin.gainaru@uni-dortmund.de.
}

nessed a surge of notions and models ${ }^{7-12}$ aimed at understanding the specificities of these liquids.

One of the problems encountered when exploring the dielectric properties of pure monohydroxy alcohols is that their Debye-like response can heavily overlap with the structural relaxation and that the latter process accounts for typically only a few percent of the total relaxation strength. This unfavorable combination of properties rendered the clear-cut assignment of, e.g., calorimetric ${ }^{13}$ observations difficult and has long hampered substantial conceptual progress in the field.

If an insufficient spectral separation of Debye-like and $\alpha$-process in monohydroxy alcohols is a concern, chemical mixing can provide a way out of this situation. In the literature, various binary, alcohol containing systems were explored ranging from the admixture of salts ${ }^{5,14}$ and nonpolar substances, ${ }^{15,16}$ which due to major differences in polarity to the alcohols often exhibit a limited mutual solubility, to the addition of other alcohols ${ }^{17}$ as well as of alkyl halides which usually circumvents that problem. Although alcohol solutions with alkyl bromides were investigated early on, ${ }^{18-20}$ and also more recently, ${ }^{21,22}$ their ability to facilitate a spectral separation of relaxation processes is systematically exploited only since a few years: Examples include mixtures of 5-methyl2-hexanol with isoamylbromide, ${ }^{23}$ of $n$-butanol (BuOH) with $n$-bromobutane $(\mathrm{BuBr}),{ }^{24,25}$ and of 2-ethyl-1-hexanol (2E1H) with $\mathrm{BuBr}^{21,26}$ In the present article we continue along these lines by investigating solutions of 2E1H with 2-ethyl-1-hexyl bromide $(2 \mathrm{E} 1 \mathrm{Br})$. In a first step, we will explore the dielectric properties of $(2 \mathrm{E} 1 \mathrm{H})_{x}(2 \mathrm{E} 1 \mathrm{Br})_{1-x}$ for the entire range of 
alcohol concentrations $x$ near and above their calorimetric glass transition temperatures $T_{g}$. These are $148 \mathrm{~K}$ for $2 \mathrm{E} 1 \mathrm{H}$ and $131 \mathrm{~K}$ for $2 \mathrm{E} 1 \mathrm{Br}^{11,27}$

For samples with intermediate alcohol concentrations we will report below that the Debye process is up to four decades slower than the structural one. We take advantage of this enormous spectral separation in such samples in order to show that their shear mechanical modulus reflects solely the structural relaxation. To this end we make use of a measuring technique which allows us to study the mechanical response over more than five decades in frequency. ${ }^{28}$ Furthermore, we employ a recent version of the Gemant-DiMarzio-Bishop model ${ }^{29-31}$ in order to clarify the interrelation of dielectric with shear mechanical properties.

Further insight can be gained from triplet state solvation dynamics experiments that were already applied to propanol, ${ }^{32}$ a short-chain monohydroxy alcohol. The dipolar probe used in the present work is sensitive to the electric modulus response of the solvent at the microscopic level. ${ }^{33}$ The results can thus be compared with shear modulus data or with the measured electric impedance represented in modulus form. ${ }^{34}$

It is not clear which process dominates the physical aging of monohydroxy alcohols, because so far only somewhat incomplete or inconclusive dielectric measurements exist: ${ }^{35-37}$ The available data do not allow one to decide whether aging takes place on the $\alpha$ time scale or on a somewhat longer scale. The currently studied equimolarly diluted alcohol is well suited to demonstrate unambiguously that physical aging is governed by the structural (or $\alpha$-) relaxation alone.

For several binary solutions of monohydroxy alcohols with alkyl bromides, such as $(\mathrm{BuOH})_{x}(\mathrm{BuBr})_{1-x}$ and $(2 \mathrm{E} 1 \mathrm{H})_{x}(\mathrm{BuBr})_{1-x}$, the occurrence of a so-called critical concentration $x_{c}$ was reported. ${ }^{24,26}$ This term is meant to imply that the shape and/or the time scale of the Debye process is more or less preserved when diluting a monohydroxy alcohol, in some cases even by $50 \%$. We address the issue of a critical concentration for $(2 \mathrm{E} 1 \mathrm{H})_{x}(2 \mathrm{E} 1 \mathrm{Br})_{1-x}$ by dielectric measurements. In addition, we use near-infrared (NIR) spectroscopy, which is sensitive to the stretching vibration of the hydroxyl group in viscous liquids, ${ }^{38}$ in order to find out whether a critical concentration is also revealed when probing the hydrogen bonds directly.

\section{EXPERIMENTAL DETAILS}

$2 \mathrm{E} 1 \mathrm{H}$ and $2 \mathrm{E} 1 \mathrm{Br}$, with stated purities of $99.6 \%$ and $95 \%$, respectively, were purchased from Sigma Aldrich and used without further treatment. After weighting and mixing, the $(2 \mathrm{E} 1 \mathrm{H})_{x}(2 \mathrm{E} 1 \mathrm{Br})_{1-x}$ systems were stored at room temperature in sealed jars for $\sim 3 \mathrm{~h}$ to achieve a good homogenization prior to any experimental investigation. No sign of phase separation was observed for the entire mixing range.

The dielectric measurements were performed using an Alpha impedance analyzer from Novocontrol that records the complex dielectric constant in a frequency range from $10^{-3} \mathrm{~Hz}$ to $10^{6} \mathrm{~Hz}$. The liquids were transferred to invar sapphire cells ${ }^{39}$ with geometrical capacitances $C_{0}$ of around $25 \mathrm{pF}$. The temperature in the cryostat was stabilized within
$0.2 \mathrm{~K}$ by a Quatro system. Dielectric aging experiments with a thermal stability of $0.02 \mathrm{~K}$ were based upon a measurement system described in Ref. 40. The shear mechanical measurements were carried out using the piezoelectric transducer technique described previously. ${ }^{41}$

NIR spectroscopy of $(2 \mathrm{E} 1 \mathrm{H})_{x}(2 \mathrm{E} 1 \mathrm{Br})_{1-x}$ mixtures was carried out at $T=300 \mathrm{~K}$ using a CARY $2300 \mathrm{UV} / \mathrm{VIS}-\mathrm{NIR}$ photospectrometer from Varian. A range of wavelengths $\lambda$ from $1000 \mathrm{~nm}$ to $2000 \mathrm{~nm}$ corresponding to wave numbers from $10000 \mathrm{~cm}^{-1}$ to $5000 \mathrm{~cm}^{-1}$ was covered with a wavelength accuracy of $0.8 \mathrm{~nm}$. The samples were placed in Hellma cuvettes (110 QX-2 mm). The dimensionless absorbance $A=\log _{10}\left(I_{0} / I\right)$ was determined from the ratio of transmitted intensity $I$ and reference intensity $I_{0}$. From all NIR spectra the absorbance of the evacuated cuvette was subtracted. This correction is not perfect since it does not account for the difference in reflection at the glass/liquid or glass/vacuum interfaces which requires prior knowledge of the sample's refractive index. The apparent negative absorbances showing up at the shortest wavelengths in NIR spectra that we show below are a consequence of this shortcoming.

For solvation dynamics experiments a small amount $\left(10^{-4} \mathrm{~mol}\right)$ of quinoxaline (QX), purified by sublimation, was dissolved in a $(2 \mathrm{E} 1 \mathrm{H})_{0.5}(2 \mathrm{E} 1 \mathrm{Br})_{0.5}$ mixture. The sample was placed in an optical cell containing UV-grade synthetic silica windows and it was vacuum sealed in a stainless steel frame. More information regarding the equipment and its properties can be found in Ref. 42. The results were obtained as described in Ref. 43, and for each temperature the $\mathrm{T}_{1} \rightarrow \mathrm{S}_{0}$ emission peak of QX was recorded $10 \mathrm{~ms}$ after the laser excitation.

\section{RESULTS AND ANALYSIS}

\section{A. Dipolar dynamics probed by dielectric spectroscopy}

In Fig. 1 we present the real and the imaginary parts of the dielectric constant for $x=0.69$ and for $x=0.31$. For the alcohol rich sample $[x=0.69$, frames (a) and (b) $]$ a twostep behavior is obvious from $\varepsilon^{\prime}(\omega)$ and correspondingly two loss maxima are seen in $\varepsilon^{\prime \prime}(\omega)$. The low-frequency relaxation, corresponding to the Debye-like process, is considerably stronger and far less broadened than the $\alpha$-relaxation which shows up at higher frequencies. For the bromide rich sample $[x=0.31$, frames (c) and (d) $]$ a two-step or two-peak pattern is revealed, again. However, here the $\alpha$-relaxation exhibits the larger amplitude. Another striking difference is seen in the overall relaxation strengths which appear almost independent of temperature for low $x$, but for large $x$ relaxation strength increases strongly upon cooling. A careful inspection of the data shows that the $\alpha$-relaxation is relatively insensitive to temperature for both concentrations, while the low-frequency dispersion gains significant strength as $T$ is lowered. The various dependences of the relaxation strengths on temperature, composition, etc. are often expressed in terms of the Kirkwood factor ${ }^{44}$ which, applied to the present data, underscores the significant differences in mutual molecular alignment associated with the $\alpha$-relaxation or with the Debye processes. 


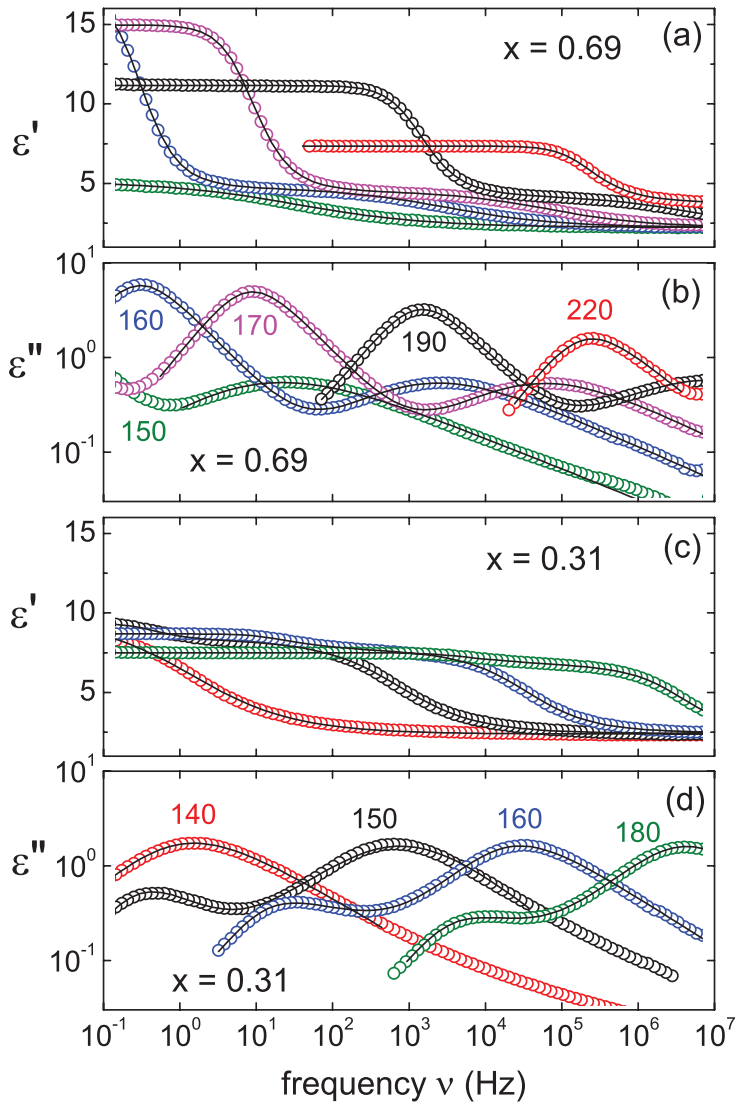

FIG. 1. Real and imaginary parts of the frequency dependent complex dielectric constant of samples with alcohol concentrations $x=0.69$ (frames a and $\mathrm{b}$ ) and $x=0.31$ (frames $\mathrm{c}$ and $\mathrm{d}$ ). The relative weights of the two relaxation processes are seen to depend strongly on temperature (given in Kelvin). The solid lines are fits using Eq. (1).

To cover the entire composition range, dielectric data were additionally recorded for $x=0,0.10,0.20,0.43,0.50$, $0.61,0.80,0.92$, and 1.00 . When compiling the dielectric loss patterns in a single plot, a smooth variation with concentration is obtained. Figure 2 presents this kind of data for $T=170 \mathrm{~K}$ and reveals that the time scales as well as the amplitudes of both processes show monotonic dependences: Starting from $2 \mathrm{E} 1 \mathrm{Br}$ one recognizes how alcohol admixture transforms the strong $\alpha$-process successively into a weak feature on the high-frequency flank of the Debye process dominating in $2 \mathrm{E} 1 \mathrm{H}$. Upon lowering $x$ the $\alpha$-peaks shift continuously to lower frequencies. This is expected since the glass transition temperature of $2 \mathrm{E} 1 \mathrm{H}\left(T_{g}=148 \mathrm{~K}\right)$ is significantly higher than that of $2 \mathrm{E} 1 \mathrm{Br}\left(T_{g}=131 \mathrm{~K}\right)$.

Focusing on the Debye (-like) peak, one can infer from Fig. 2 that a dilution of $2 \mathrm{E} 1 \mathrm{H}$ reduces the strength of this process considerably. Even in the limit $x \rightarrow 0$ a small remnant of this relaxational feature appears to survive. We will report on this issue in more detail elsewhere and here point out only that the smooth evolution of this low-frequency process suggests a common microscopic origin for all compositions.

A more quantitative analysis of the dielectric data can be achieved by describing them using a suitable fitting function. The Debye-like dielectric loss can develop an asymmetric shape in diluted alcohols and in the current article is thus parameterized in terms of a Cole-Davidson function with a

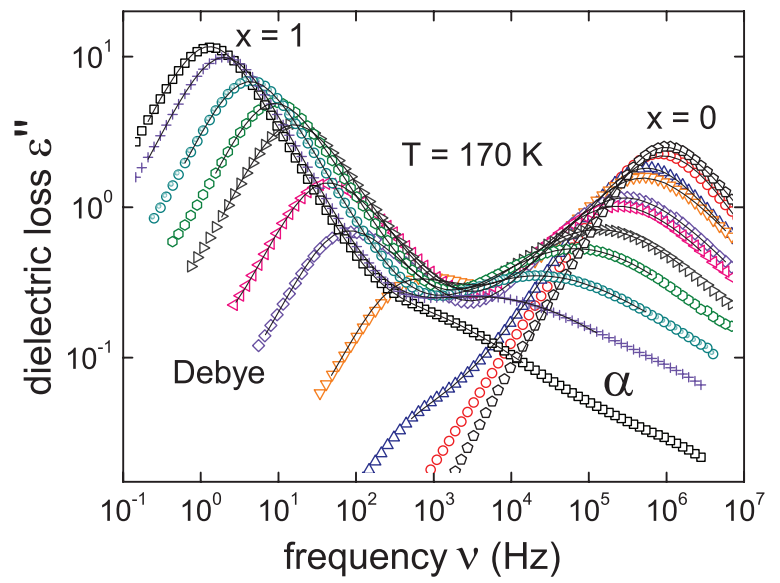

FIG. 2. Dielectric loss spectra of $(2 \mathrm{E} 1 \mathrm{H})_{x}(2 \mathrm{E} 1 \mathrm{Br})_{1-x}$ evolving smoothly for $x=0,0.10,0.20,0.31,0.43,0.50,0.61,0.69,0.80,0.92$, and 1.00 so that only the pure liquids needed to be labeled in the figure. For large $x$ the Debyelike response is most prominent, while for $x=0$ the structural relaxation dominates at the given temperature of $170 \mathrm{~K}$. The solid lines represent fits using Eq. (1).

broadening coefficient $\gamma_{D}$. The $\alpha$-process is often found to be symmetrically broadened in monohydroxy alcohols ${ }^{24,45}$ (see also Fig. 1), in contrast to the behavior of most other supercooled liquids. We describe the present data with a Cole-Cole function in which $\alpha_{\alpha}$ is a measure for the degree of broadening. As in previous studies ${ }^{24,25}$ we thus employ a superposition of two relaxation processes

$$
\varepsilon^{*}(\nu)=\varepsilon_{\infty}+\frac{\Delta \varepsilon_{D}}{\left(1+2 \pi i v \tau_{D}\right)^{\gamma_{D}}}+\frac{\Delta \varepsilon_{\alpha}}{1+\left(2 \pi i v \tau_{\alpha}\right)^{\alpha_{\alpha}}} .
$$

Here $\varepsilon_{\infty}$ designates the permittivity at high frequencies, while $\Delta \varepsilon_{\alpha}$ and $\Delta \varepsilon_{D}$ are the relaxation strengths of the $\alpha$ - and of the Debye-like processes, respectively. The corresponding relaxation times are called $\tau_{D}$ and $\tau_{\alpha}$.

The solid lines in Figs. 1 and 2, calculated using Eq. (1), are seen to provide very good fits to the dielectric data. Similar fits were achieved for other concentrations and temperatures. The relaxation times and relaxation strengths thus obtained as a function of the alcohol concentration are summarized in Fig. 3. Figure 3(a) presents $\tau_{D}$ and in Fig. 3(b) the $\alpha$-relaxation times $\tau_{\alpha}$ are shown for several temperatures. In order to emphasize the common features in the concentration dependence, all the data are normalized to their values at $x=1$. The already mentioned monotonic increase of $\tau_{D}$ and $\tau_{\alpha}$ with $x$ is nicely borne out by Fig. 3. More strikingly, this plot demonstrates that two composition regimes can be distinguished: Below $x \sim 0.5$ the logarithm of $\tau_{D}$ follows a stronger linear dependence than above $x \sim 0.6$, while for $\log \left(\tau_{\alpha}\right)$ the dependence for $x>0.6$ is stronger than it is for lower $x$.

Consequently, the ratio of the two time scales is largest for $x=0.5-0.6$. This statement can be quantified on the basis of the decoupling ratio ${ }^{46}$

$$
d(x)=\log _{10} \frac{\tau_{D}(x)}{\tau_{\alpha}(x)} .
$$

The inset of Fig. 3 shows indeed a broad $d(x)$ maximum near $x=0.6$ indicative for a decoupling of time scales of 


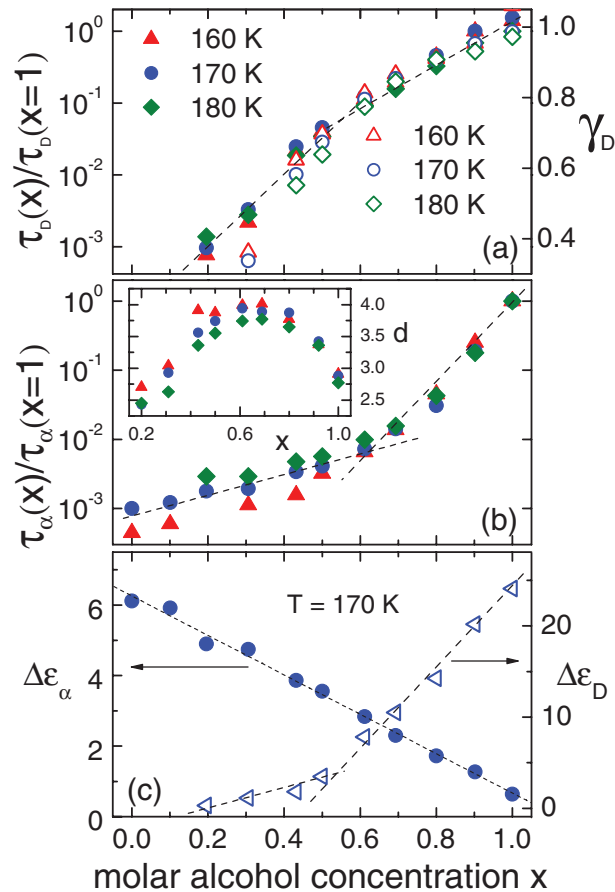

FIG. 3. The concentration dependence of the relaxation times $\tau_{D}(x)$ and $\tau_{\alpha}(x)$ as scaled to their values for $x=1$ are plotted as full symbols in frames (a) and (b) for several temperatures. The solid lines are drawn to emphasize the occurrence of a so called critical concentration $x_{C}$. In frame (a) the ColeDavidson parameter $\gamma_{D}$, describing the shape of the Debye-like relaxation, is also given as open symbols. It follows the same trend as the time constants, hence also hints at $x_{C}$. The inset in frame (b) displays the decoupling index, $d(x)=\log _{10}\left[\tau_{D}(x) / \tau_{\alpha}(x)\right]$, as defined in Eq. (2). It demonstrates that $\tau_{D}(x)$ and $\tau_{\alpha}(x)$ may differ by up to about four decades.

about four decades. Such a tremendous separation and thus the clear-cut resolution of Debye-like and $\alpha$-relaxation devoid of a significant spectral overlap facilitates the assignment and interpretation of results obtained for roughly equimolar mixtures of $2 \mathrm{E} 1 \mathrm{H}$ with $2 \mathrm{E} 1 \mathrm{Br}$. This will be exploited in the shear mechanical, physical aging, and solvation measurements that are presented in Sec. III C-III E, respectively.

Further evidence for a change of behavior near $x \sim 0.5-0.6$ comes from the composition dependence of the Cole-Davidson exponent $\gamma_{D}$ which relates to the Debye-like process, see Fig. 3(a). For $x \sim 0.5-0.6$ both the $\alpha$ - and the Debye-like relaxations are about equally strong, at least for $T=170 \mathrm{~K}$, see Fig. 2. Figure 3(c) documents that $\Delta \varepsilon_{D}$ shows a crossover at $x \sim 0.5$. On the other hand, the spectral width (not shown) and the relaxation strength of the $\alpha$ relaxation [see Fig. 3(c)] interpolate linearly between those of the pure substances without a sign of a peculiar behavior near $x \sim 0.5-0.6$.

\section{B. Hydrogen bond populations probed by near-infrared spectroscopy}

In Fig. 4 NIR spectra of $(2 \mathrm{E} 1 \mathrm{H})_{x}(2 \mathrm{E} 1 \mathrm{Br})_{1-x}$ are shown for wavelengths ranging from about $1330 \mathrm{~nm}$ to about 1680 nm. All spectra were recorded at $T=300 \mathrm{~K}$ and were corrected as detailed in Sec. II. For neat $2 \mathrm{E} 1 \mathrm{Br}(x=0$, lowermost curve) a series of bands is seen to exist. These bands

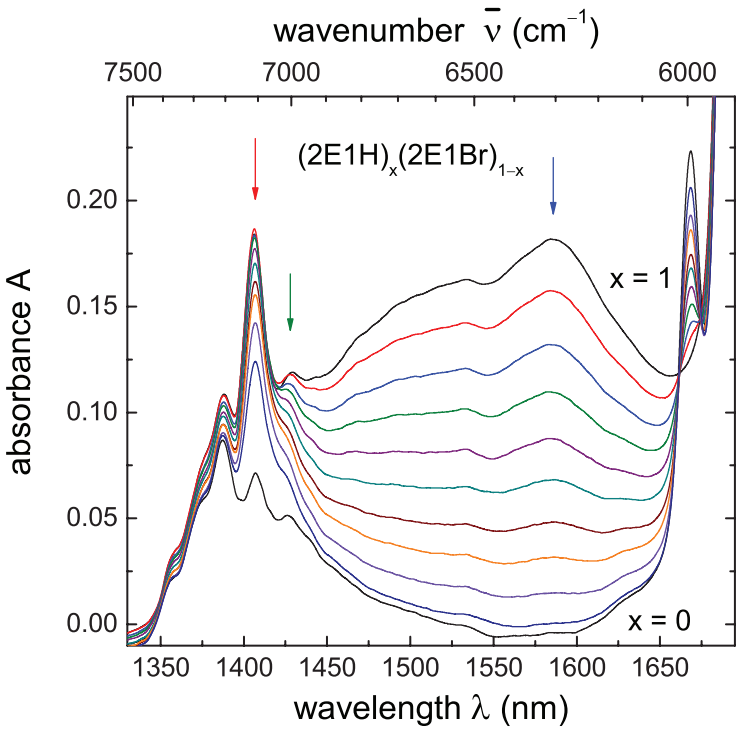

FIG. 4. NIR spectra for $(2 \mathrm{E} 1 \mathrm{H})_{x}(2 \mathrm{E} 1 \mathrm{Br})_{1-x}$ focusing on the spectral range in which $\mathrm{OH}$ overtones appear. The arrows highlight the approximate positions of the maxima corresponding to the so called monomer band $(\lambda \approx 1410 \mathrm{~nm})$, the terminal band $(\lambda \approx 1430 \mathrm{~nm})$, and the polymer band $(\lambda \approx 1590 \mathrm{~nm})$.

are assigned to combinations of stretching and bending vibrations involving carbon and hydrogen atoms ${ }^{47}$ that will be termed $\mathrm{CH}_{n}$ bands in the following. The sharp feature around $6000 \mathrm{~cm}^{-1}$ is a combination band involving the $\alpha-\mathrm{CH}_{2}$ groups that are located close to the bromide atom. ${ }^{47-49}$ Hence, the intensity of this band decreases linearly with increasing alcohol concentration.

Furthermore, with increasing $x$, additional bands appear in the spectra that are due to the first overtones of the $\mathrm{OH}$ stretching vibrations. The assignment of these bands (cf. the arrows in Fig. 4) is illustrated in Fig. 5(b). The band around $1410 \mathrm{~nm}$ corresponds to $\mathrm{OH}$ species for which the protons are not involved in hydrogen bonds and will be called in the following the "monomer" band (free $\mathrm{OH}$ group, so-called $\alpha$ state, to be distinguished from the terminal $\mathrm{OH}$ group, called $\beta$-state, according to the nomenclature of Graener et al. ${ }^{50}$ ). Since, usually, the two species cannot be easily spectroscopically differentiated they are commonly, though not completely unambiguously so, called the "monomer" band. The small absorbance feature near $1430 \mathrm{~nm}$, termed here as the terminal band, reflects the vibration of that terminal $\mathrm{OH}$ group which is hydrogen bonded via its proton ( $\gamma$-state in Ref. 50). The broad peak around $1590 \mathrm{~nm}$ is due to strongly hydrogen bonded non-terminal $\mathrm{OH}$ groups and will be termed in the following the polymer band ( $\delta$-state according to Ref. 50). At frequencies between these latter two bands, other species like cyclic or branched aggregates exist. The assignment made here is in harmony with other (N)IR spectroscopy studies. ${ }^{51-53}$

As $2 \mathrm{E} 1 \mathrm{H}$ contains many $\mathrm{CH}_{n}$ groups, the $\mathrm{OH}$ overtone region is strongly overlapped with hydrocarbon combination bands. ${ }^{47,54}$ Since the contribution of the $\mathrm{CH}_{n}$ groups is practically independent of $x$, we calculated difference spectra, $\Delta A(\lambda, \mathrm{x})=A(\lambda, x)-A(\lambda, 0)$, by subtracting the spectrum of neat $2 \mathrm{E} 1 \mathrm{Br}$. The resulting differential absorbances $\Delta A_{\text {mono }}$, $\Delta A_{\text {term }}$, and $\Delta A_{\text {poly }}$ of the difference spectra (not shown) 


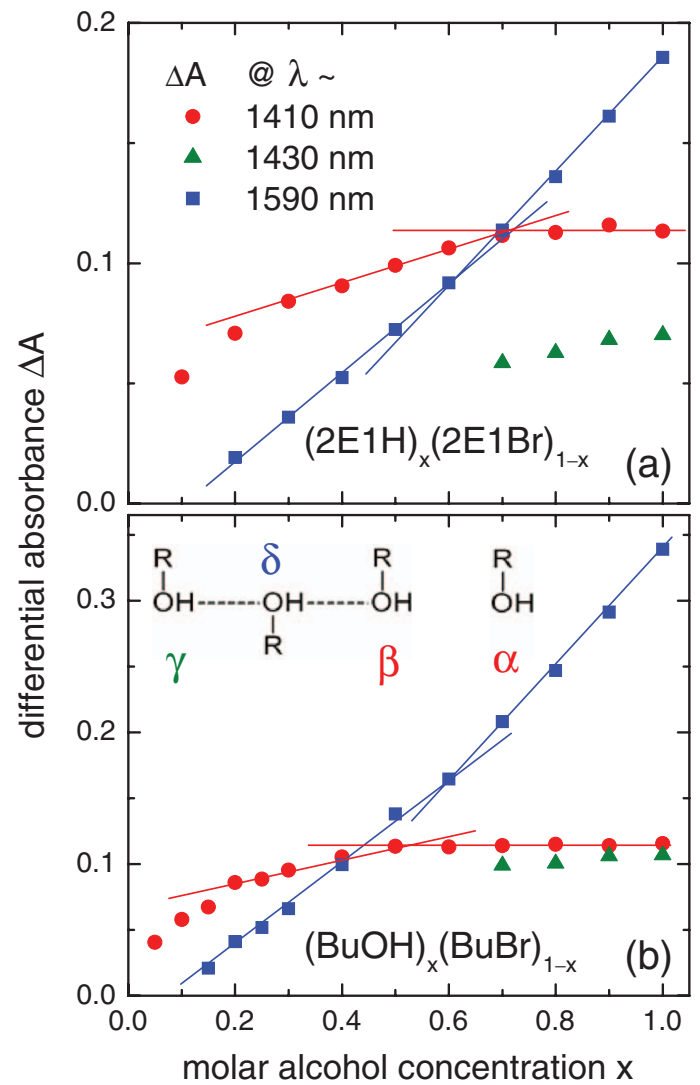

FIG. 5. (a) Differential band intensities $\Delta A$ for $(2 \mathrm{E} 1 \mathrm{H})_{x}(2 \mathrm{E} 1 \mathrm{Br})_{1-x}$ read out near the wavelengths marked by the arrows in Fig. 4. (b) Analogous plot for $(\mathrm{BuOH})_{x}(\mathrm{BuBr})_{1-x}$. Here the "monomer," the terminal, and the polymer bands appear at wavelengths similar to those for $(2 \mathrm{E} 1 \mathrm{H})_{x}(2 \mathrm{E} 1 \mathrm{Br})_{1-x}$. The lines highlight the piecewise linear concentration dependences of the band intensities. The sketches of the molecular structures in frame (b) illustrate the nomenclature of Graener et al. ${ }^{50}$ used in the assignment of $\mathrm{OH}$ bands.

read out at the positions of the "monomer," terminal, and polymer bands, respectively, are compiled as a function of $x$ in Fig. 5(a).

As can be seen for the polymer band, the differential absorbance decreases almost linearly with decreasing alcohol concentration $x$. As emphasized by the lines in Fig. 5(a), the slope in $\Delta A_{\text {poly }}(x)$ changes around $x \sim 0.6$. A change in behavior is also featured by the monomer band. In the alcohol rich region, $\Delta A_{\text {mono }}(x)$ is practically constant, whereas for $x<0.6$ it starts to become smaller. The differential absorbance for the terminal band can be read out reliably for $x \geq 0.7$ only. Below this concentration the terminal band is not clearly resolved but rather appears as a shoulder on the large-wavelength flank of the "monomer" band.

Since, starting from neat $2 \mathrm{E} 1 \mathrm{H}(x=1)$ the number of free oscillators (approximately given by the monomer band intensity) does not change and the terminal band intensity slightly decreases, the number of hydrogen-bonded structures basically stays constant down to $x \approx 0.6$. Therefore, the strong decrease of the polymer band observed here must be due to a decrease in the size of the supramolecular structures. For $x<0.6$ no conclusions can be drawn, as the contribution of the terminal band cannot be further resolved. The monomer

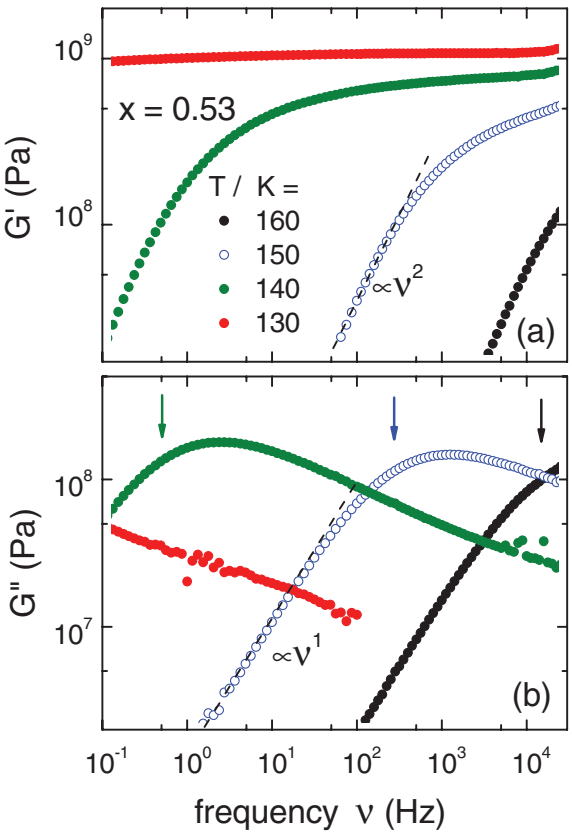

FIG. 6. (a) Real part and (b) imaginary part of the frequency dependent shear modulus of $(2 \mathrm{E} 1 \mathrm{H})_{0.53}(2 \mathrm{E} 1 \mathrm{Br})_{0.47}$ measured for several temperatures. The arrows mark the frequency positions of the dielectric loss peaks corresponding to the structural relaxation. The dashed lines highlight the power laws characterizing the low-frequency response of "simple" liquids.

band seems to hint at another critical concentration around $x$ $\approx 0.25$ as the slope in $\Delta A_{\text {mon }}(x)$ changes once again.

To check our interpretation $(\mathrm{BuOH})_{x}(\mathrm{BuBr})_{1-x}$, a similar binary system was studied [see Fig. 5(b)]. Here, the same behavior of the differential absorbance is observed: the intensity of "monomer" band, as that of the terminal band, is essentially constant above an alcohol concentration of 0.5 . The same critical butanol concentration was previously identified by dielectric spectroscopy. ${ }^{24}$ The similarity of the NIR results obtained for both the $2 \mathrm{E} 1 \mathrm{H}$ and the $\mathrm{BuOH}$ mixtures indicates that the alcohol molecules, added to solutions above the critical concentration, become part of the existing hydrogen bonded structures and increase the size (and not the number) of these objects.

\section{Frequency dependent shear response}

In order to test the relationship of dielectric and viscoelastic properties in detail we performed measurements of the complex shear modulus $G^{*}(v)=G^{\prime}(v)+i G^{\prime \prime}(v)$ for frequencies ranging from $0.1 \mathrm{~Hz}$ to about $10 \mathrm{kHz}$. For a sample with $x=0.53$ the real part, $G^{\prime}(v)$, and the imaginary part, $G^{\prime \prime}(v)$, are shown in Figs. 6(a) and 6(b), respectively. A high-frequency shear modulus of $G_{\infty} \approx 1 \mathrm{GPa}$ can be read off from the data in Fig. 6(a) at the lowest temperature. In Fig. 6(b) several well resolved mechanical loss maxima show up. The arrows included in this figure indicate the positions of the dielectric loss peaks (corresponding to the $\alpha$-process) of the same sample. It becomes clear that the mechanical loss peaks appear at frequencies which are $\tau_{\text {diel }} / \tau_{\text {shear }} \approx 5$ times larger than the $\varepsilon^{\prime \prime}(\nu)$ peak frequencies that correspond to the $\alpha$-relaxation. Differing peak 


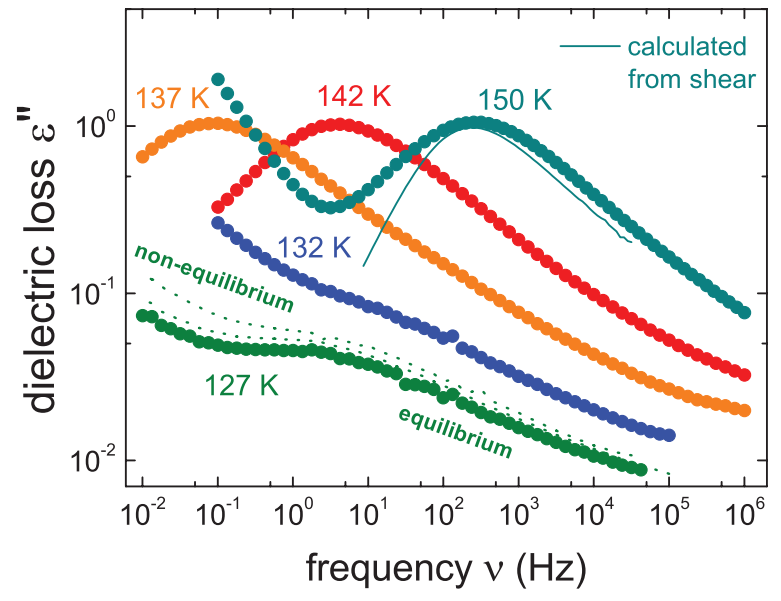

FIG. 7. Dielectric loss spectra of $(2 \mathrm{E} 1 \mathrm{H})_{0.5}(2 \mathrm{E} 1 \mathrm{Br})_{0.5}$ as measured in equilibrium (filled symbols) and under non-equilibrium conditions (dotted lines). The latter were recorded about 3 and $300 \mathrm{~min}$ after stepping the temperature from 132 to $127 \mathrm{~K}$. The solid line is calculated from the complex shear modulus according to Eq. (3) and the parameters given below that equation. The peak frequencies of the measured and the calculated dielectric losses agree nicely while the spectral shapes differ somewhat.

frequencies are typical when comparing (dielectric) susceptibilities with (mechanical) moduli. ${ }^{55,56}$ The shear data clearly demonstrate that a terminal relaxation mode is experimentally accessed in the investigated frequency window: In Fig. 6 the dashed lines indicate that in the low-frequency range the $v$ dependences of $G^{\prime}(v)$ and $G^{\prime \prime}(v)$ for $T=150 \mathrm{~K}$ become identical with those expected for "simple" liquids (within the Maxwell model), i.e., $G^{\prime}(v) \propto v^{2}$ and $G^{\prime \prime}(v) \propto v{ }^{57}$ In particular, for the data recorded at $160 \mathrm{~K}$ and $150 \mathrm{~K}$ it is evident that the Debye process in $(2 \mathrm{E} 1 \mathrm{H})_{0.53}(2 \mathrm{E} 1 \mathrm{Br})_{0.47}$ does not leave any measurable trace in the mechanical response above the resolution limit $\left(10^{7} \mathrm{~Pa}\right)$ available in the present work. This result is compatible with previous conclusions drawn for pure $2 \mathrm{E} 1 \mathrm{H}^{28}$

A more detailed comparison of dielectric and shear mechanical responses, which allows one to check the compatibility of the peak maxima and in favorable cases also of the entire spectral shapes of $G^{*}(\nu)$ and $\varepsilon^{*}(\nu)$, is possible on the basis of a simple model. ${ }^{29}$ In its recently refined form this approach yields for the dielectric loss ${ }^{31}$

$$
\varepsilon^{\prime \prime}(v)=\frac{\Lambda B^{2}(\Lambda) G^{\prime \prime}(v)}{\left[1+\Lambda B(\Lambda) G^{\prime}(\nu)\right]^{2}+\left[\Lambda B(\Lambda) G^{\prime \prime}(\nu)\right]^{2}}
$$

with the abbreviation $B(\Lambda)=\frac{1}{2}\left[\Delta \varepsilon_{\alpha}+\sqrt{\Delta \varepsilon_{\alpha}^{2}+4 \Delta \varepsilon_{\alpha} /\left(\Lambda G_{\infty}\right)}\right]$ and $\Lambda$ denoting the electro-viscoelastic constant which is considered a free parameter. We calculated $\varepsilon^{\prime \prime}(\nu)$ from $G^{*}(\nu)$ on the basis of Eq. (3) with $\Lambda=7 \times 10^{-10} \mathrm{~Pa}^{-1}$ and present the results of this procedure as lines in Fig. 7 for $T=150 \mathrm{~K}$ (for which dielectric and rheological data are available). Excellent agreement of the calculated with the measured loss peak frequency and loss maximum is obtained, while the model underestimates the dielectric loss peak width somewhat. This way of analyzing the data confirms the correspondence of the mechanical response to the $\alpha$-relaxation.

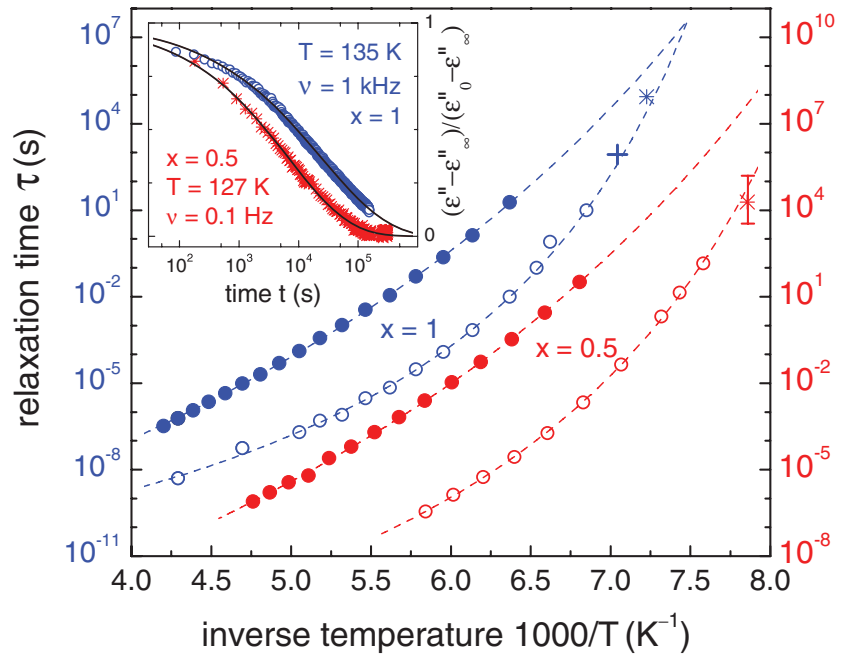

FIG. 8. Arrhenius plot of $2 \mathrm{E} 1 \mathrm{H}$ (left ordinate axis) and of $(2 \mathrm{E} 1 \mathrm{H})_{0.5}(2 \mathrm{E} 1 \mathrm{Br})_{0.5}$ (right ordinate axis). The full and the open circles represent $\tau_{D}$ and $\tau_{\alpha}$, respectively, from dielectric measurements. The lines are fits using the Vogel-Fulcher law, Eq. (4), with the parameters given below that equation. The stars represent time constants from aging, $\left\langle\tau_{\text {age }}\right\rangle$, determined from the isothermal dielectric loss curves shown in the inset. The error bar indicates the variation limits for $\left\langle\tau_{\text {age }}\right\rangle$ between the equilibrium (linear) relaxation times at the temperatures before and after the $T$-jump, $\tau_{\mathrm{i}}(T=129.5 \mathrm{~K})<\left\langle\tau_{\text {age }}\right\rangle<\tau_{\mathrm{f}}(T=127 \mathrm{~K})$ calculated based on Eq. (4). The time scale taken from a previous NIR detected temperature jump experiment on pure $2 \mathrm{E} 1 \mathrm{H}$ (Ref. 60) is marked by a cross.

\section{Physical aging}

In Fig. 7 we show several dielectric spectra for $(2 \mathrm{E} 1 \mathrm{H})_{0.5}(2 \mathrm{E} 1 \mathrm{Br})_{0.5}$, a sample for which the time scale of the $\alpha$-process reaches $100 \mathrm{~s}$ near $T_{g, \alpha} \approx 132 \mathrm{~K}$. Reducing the temperature in steps of $5 \mathrm{~K}$ and starting from $152 \mathrm{~K}$, equilibration of the loss spectra is attained practically instantaneously, except at the lowest temperature. Thermal stability of about $\pm 0.02 \mathrm{~K}$ was typically reached within a time interval of $200 \mathrm{~s}$. The immediate equilibration for all temperatures $T>130 \mathrm{~K}$, which was confirmed by repeat runs at longer times, rules out that aging processes take place on the scale of $\tau_{D}$. This confirms a previous report on pure $2 \mathrm{E} 1 \mathrm{H}$ which, however, was unable to resolve the exact time scale of this physical aging process. ${ }^{36}$

At a base temperature of $127 \mathrm{~K}$, which is about $5 \mathrm{~K}$ below $T_{g, \alpha}$, the loss data exhibit a pronounced shoulder in the frequency range of about 1 to $10 \mathrm{~Hz}$ which after waiting for about 1 day evolves into a well resolved secondary relaxation peak. This is due to the fact that at a given frequency the dielectric loss contribution arising from the structural relaxation successively decreases with time. After a jump from $129.5 \mathrm{~K}$ to $127 \mathrm{~K}$, we monitored this reduction of $\varepsilon^{\prime \prime}$ for a frequency of $0.1 \mathrm{~Hz}$ in a time range from about $2 \times 10^{2}$ to about $3 \times 10^{5} \mathrm{~s}$ and present our results in the inset of Fig. 8. One recognizes that at the longest times structural equilibrium is fully established. For the sake of simplicity the aging curve can be described using a Kohlrausch function $\varepsilon^{\prime \prime}(T) \propto \exp \left[-\left(t / \tau_{\text {age }}\right)^{\beta_{\text {age }}}\right]$. From a least-squares fit to the data we obtain an aging time $\tau_{\text {age }}=6.7 \times 10^{3} \mathrm{~s}$ and the corresponding stretching exponent is $\beta_{\text {age }}=0.42$ yielding a mean 
aging time of $\left\langle\tau_{\text {age }}\right\rangle=\tau_{\text {age }} \Gamma\left(1 / \beta_{\text {age }}\right) / \beta_{\text {age }} \approx 2 \times 10^{4} \mathrm{~s}$ with $\Gamma$ denoting Euler's Gamma function.

A slightly less complete set of data with re-equilibrating times extending only up to about $1.6 \times 10^{5} \mathrm{~s}$ was recorded for neat $2 \mathrm{E} 1 \mathrm{H}$ subsequent to stepping the temperature from 138 to $135 \mathrm{~K}$. The aging time dependent dielectric loss is also shown in the inset of Fig. 8. Here a fit using a Kohlrausch function yields $\tau_{\text {age }}=2.4 \times 10^{4} \mathrm{~s}$ and $\beta_{\text {age }}=0.38$ leading to $\left\langle\tau_{\text {age }}\right\rangle \approx 8.7 \times 10^{4}$ s.

In Fig. 8 the aging times $\left\langle\tau_{\text {age }}\right\rangle$ are compared with other dielectric relaxation times, $\tau_{D}$ and $\tau_{\alpha}$, collected for $2 \mathrm{E} 1 \mathrm{H}$ and for $(2 \mathrm{E} 1 \mathrm{H})_{0.5}(2 \mathrm{E} 1 \mathrm{Br})_{0.5}$. As shown by the dotted lines, the $\tau_{D}$ and the $\tau_{\alpha}$ times can be parameterized by a Vogel-FulcherTammann law $^{58}$ that is typically used to describe the temperature dependence of the relaxation times ${ }^{59}$ for molecular supercooled liquids,

$$
\tau=\tau_{0} \exp \left[B /\left(T-T_{0}\right)\right] .
$$

Here $\tau_{0}^{-1}$ designates an attempt frequency and the coefficients $B$ and $T_{0}$ determine the form of the $\tau(T)$ curves. The parameters for $x=0.5$ are $\tau_{0, D}=1.7 \times 10^{-15} \mathrm{~s}, \tau_{0, \alpha}=1.3 \times 10^{-14}$ $\mathrm{s}, B_{D}=2700 \mathrm{~K}, B_{\alpha}=1250 \mathrm{~K}, T_{0, \mathrm{D}}=75 \mathrm{~K}$, and $T_{0, \alpha}=98 \mathrm{~K}$. For $x=1$ the corresponding parameters were previously reported in Ref. 60.

Several features are important to note from Fig. 8: (i) Although $\tau_{D}$ and $\tau_{\alpha}$ display a tendency to merge towards low temperatures, they remain separated by more than three decades near $T_{g, \alpha}$ for $x=0.5$. (ii) For $x=1$ the aging time $\left\langle\tau_{\text {age }}\right\rangle$ is intermediate between $\tau_{\alpha}$ and (the extrapolated) $\tau_{D}$ which at $135 \mathrm{~K}$ are in close proximity. (iii) For $x=0.5$, however, $\left\langle\tau_{\text {age }}\right\rangle$ is fully compatible with the extrapolation of $\tau_{\alpha}$ but not with that of $\tau_{D}$, demonstrating that the $\alpha$-process governs the physical aging, also in monohydroxy alcohols. Hence, it is fully justified to call the $\alpha$-process the structural relaxation. (iv) In the inset one can observe that for $x=0.5$ the aging curve reaches its saturation (i.e., the system becomes fully equilibrated) for a waiting time that is slightly above $10^{5} \mathrm{~s}$, which is still considerably below the extrapolated value for $\tau_{D}\left(\approx 10^{8} \mathrm{~s}\right)$ at this temperature. This implies that the conclusion drawn in item (iii) remains valid independent of the approach used for estimating $\left\langle\tau_{\text {age }}\right\rangle$.

\section{E. Solvation dynamics}

$\mathrm{T}_{1} \rightarrow \mathrm{S}_{0}$ emission spectra of QX in $(2 \mathrm{E} 1 \mathrm{H})_{0.5}(2 \mathrm{E} 1 \mathrm{Br})_{0.5}$ were recorded for several temperatures between 112 and $152 \mathrm{~K}$ using a fixed acquisition time of $10 \mathrm{~ms}$. This is significantly shorter than the phosphorescence lifetime of QX that is about $0.3 \mathrm{~s}$. In order to determine the average emission energy $\left\langle v_{e}\right\rangle$ each emitted spectrum was interpolated by a Gaussian function. The temperature dependence thus obtained for $\left\langle v_{e}\right\rangle$ is plotted in Fig. 9.

At temperatures below $130 \mathrm{~K}$ the average emission energy, $\left\langle v_{e}\right\rangle_{0}$, is virtually temperature invariant. This indicates that the solvent dynamics is frozen in on the time scale of our isochronal experiment. For temperatures above about $130 \mathrm{~K}$, a decrease of $\left\langle v_{e}\right\rangle$ below $\left\langle v_{e}\right\rangle_{0}$ can be noticed. This continuous variation of the Stokes shift signals that the time scale

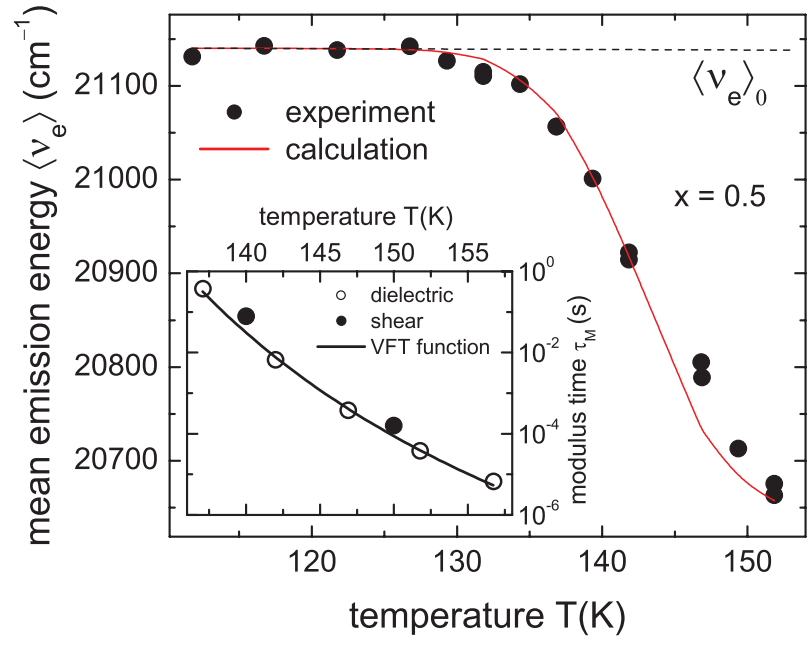

FIG. 9. The dots represent the temperature dependence of the average emission energy $\left\langle v_{e}\right\rangle$ (in units of $\mathrm{cm}^{-1}$ ) of $10^{-4} \mathrm{~mol} \mathrm{QX}$ in $(2 \mathrm{E} 1 \mathrm{H})_{0.5}(2 \mathrm{E} 1 \mathrm{Br})_{0.5}$ recorded $10 \mathrm{~ms}$ after laser excitation. The dashed line marks the lowtemperature plateau $\left\langle v_{e}\right\rangle_{0}$. The solid line is calculated from Eq. (6) using the modulus time scale $\tau_{M}$ represented in the inset as solid line and with the parameters given in the text. The plot in the inset also shows the times from the dielectric and from the shear modulus.

of the polarization fluctuation in the local environment of QX decreases towards the value of the experimental time window $(10 \mathrm{~ms})$. Previous solvation dynamics investigations demonstrated that, for various chromophore/solvent combinations, this redshift $\left(\left\langle v_{e}\right\rangle-\left\langle v_{e}\right\rangle_{0}<0\right)$ occurs close to the glass transition temperature of the molecular solvent. ${ }^{61}$ Indeed, the onset in the decrease of the emission energy $\left\langle v_{e}\right\rangle$ measured for $(2 \mathrm{E} 1 \mathrm{H})_{0.5}(2 \mathrm{E} 1 \mathrm{Br})_{0.5}$ (see Fig. 9) is very close to its $T_{g, \alpha}(\approx 132 \mathrm{~K})$.

In order to quantify the temperature evolution of the solvent dynamics we follow previous studies ${ }^{61,62}$ and consider the Stokes shift correlation function,

$$
C(t, T)=\frac{\left\langle v_{e}\right\rangle(t, T)-\left\langle v_{e}\right\rangle_{\infty}}{\left\langle v_{e}\right\rangle_{0}-\left\langle v_{e}\right\rangle_{\infty}},
$$

in the form of a Kohlrausch stretched exponential, $C(t, T)=\exp \left\{-[t / \tau(T)]^{\beta(T)}\right\}$. Using this expression the temperature variance of the energy shift can be written as

$$
\left\langle v_{e}\right\rangle(T)=\left\langle v_{e}\right\rangle_{\infty}+\left(\left\langle v_{e}\right\rangle_{0}-\left\langle v_{e}\right\rangle_{\infty}\right) \exp \left\{-\left[t_{0} / \tau(T)\right]^{\beta(T)}\right\}
$$

with $t_{0}=10 \mathrm{~ms}$ denoting the experimental time scale.

The non-equilibrium polarization fluctuations of the mixture solvent are caused by a change in the electronic charge distribution (rather than in the electrical field) of the excited chromophore. Therefore, the solvation experiment probes an electric modulus response (instead of a susceptibility). ${ }^{32}$ In this sense, it is appropriate to compare the solvation results with the ones obtained via dielectric spectroscopy after converting the permittivity $\varepsilon^{*}$ data to the electric modulus $M^{*}=1 / \varepsilon^{*}$. Accordingly, $\tau$ and $\beta$ in Eq. (6) have to be replaced by $\tau_{\mathrm{M}}$ and $\beta_{\mathrm{M}}$ that are specific for this quantity.

To this end the dielectric data of $(2 \mathrm{E} 1 \mathrm{H})_{0.5}(2 \mathrm{E} 1 \mathrm{Br})_{0.5}$ was transformed to the modulus representation and for every temperature a modulus time scale was extracted as $\tau_{M}=1 /\left(2 \pi v_{M}\right)$. Here $v_{M}$ is the peak frequency in the 
imaginary part, $M^{\prime \prime}(v)$, that corresponds to the $\alpha$-process. The results for $\tau_{\mathrm{M}}(T)$, as shown in the inset of Fig. 9, were parameterized using Eq. (4) and yielded $\tau_{0}=5 \times 10^{-15} \mathrm{~s}$, $B=1200 \mathrm{~K}$, and $T_{0}=102 \mathrm{~K}$. Regarding the stretching parameter, the imaginary part of the modulus could be interpolated well by the Laplace transform of a Kohlrausch function and yielded $\beta_{\mathrm{M}}=0.33$, independent of the temperature in the range in which the solvation dynamics was monitored.

With these parameters at hand, $\left\langle v_{e}\right\rangle(T)$ can be calculated according to Eq. (6). In this expression, only one parameter, $\left\langle v_{e}\right\rangle_{\infty}$, is not already fixed by experiment. Using $\left\langle v_{e}\right\rangle_{\infty}=20$ $650 \mathrm{~cm}^{-1}$, it turns out that the calculation provides a good description of the energy shift probed in our solvation experiments, cf. Fig. 9. It should be emphasized that the modulus response that enters into these calculations corresponds to the structural relaxation.

\section{DISCUSSION}

The time scale derived from the aging experiments is in excellent agreement with the structural relaxation times as documented for a sample with $x=0.5$ in Fig. 8. Nevertheless, this agreement deserves comment because we compare results from dielectric linear-response experiments with those induced by a sizeable temperature step. By their very nature the latter experiments are nonlinear and this property applies also to aging measurements. Several methods have been devised for their description. ${ }^{63-66}$ For analyzing the present data, we simply quantify an aging time scale, $\tau_{\text {age }}$, based upon the stretched exponential decay outlined above. Any model of physical aging will predict that $\tau_{\text {age }}$ obtained in such a manner has to be intermediate between the equilibrium (linear) relaxation times at the temperatures before and after the $T$-jump, $\tau_{\mathrm{i}}(T=129.5 \mathrm{~K})<\tau_{\text {age }}<\tau_{\mathrm{f}}(T=127 \mathrm{~K})$. This range is represented by an "error" bar in Fig. 8 and clearly demonstrates that $\tau_{\alpha}$ rather than $\tau_{\mathrm{D}}$ governs the process of physical aging.

It is also worthwhile to compare the present physical aging data with previous ones that were acquired using dielectric spectroscopy ${ }^{35,36}$ or NIR spectroscopy. ${ }^{60}$ The NIR data specifically monitored the equilibration of the hydrogen-bond population of pure $2 \mathrm{E} 1 \mathrm{H}$ and showed that it proceeds on the time scale of the $\alpha$-process (see the cross in Fig. 8). Within the limits of the signal-to-noise ratio in those experiments reliable statements could not be made regarding the stretching of the re-equilibration curve. The present dielectric aging experiments on $2 \mathrm{E} 1 \mathrm{H}$ which are not selectively sensitive to the hydrogen bond dynamics were performed at lower temperatures than the NIR measurements. Here, about $13 \mathrm{~K}$ below $T_{g, \alpha}, \tau_{D} / \tau_{\alpha} \approx 10$ and the association of $\left\langle\tau_{\text {age }}\right\rangle$ to either process is far from ambiguous. In a previous dielectric aging study an exponential aging curve was reported for the pure monohydroxy alcohol 5-methyl-2-hexanol. ${ }^{35}$ At the temperature of $131.4 \mathrm{~K}$ (i.e., $16.7 \mathrm{~K}$ below its $T_{g}$ of $148.1 \mathrm{~K}$ ) at which these experiments were performed $\tau_{D} / \tau_{\alpha}$ is estimated to be very close to or even below 1, see Fig. 3 of Ref. 67.

For $(2 \mathrm{E} 1 \mathrm{H})_{0.5}(2 \mathrm{E} 1 \mathrm{Br})_{0.5}$ the situation is different: At $127 \mathrm{~K}$, the base temperature for the aging experiment, the extrapolated $\tau_{D} / \tau_{\alpha}$ is about three decades and clearly physical aging takes place on the time scale of the $\alpha$-relaxation and not on that of the Debye-like process. Therefore, the notion that the structural relaxation could contribute to both processes, as recently suggested, ${ }^{68}$ is not supported by the present data, unless this is meant to say that the $\alpha$-relaxation represents the elementary step of the Debye-like process. The latter implication is part, e.g., of the transient chain model. ${ }^{8}$

Our aging study provides just one example of the utility of chemical mixing for the spectral separation of various processes and this was also exploited in the present shear mechanical experiment. Different from a related previous investigation, ${ }^{28}$ for $(2 \mathrm{E} 1 \mathrm{H})_{0.5}(2 \mathrm{E} 1 \mathrm{Br})_{0.5}$ we reached the simple liquid limit [i.e., $G^{\prime}(v) \propto v^{2}$ and $G^{\prime}(v) \propto v$ at low frequencies] since the slowest elastic mode is obviously present in the investigated frequency range. The onset of this terminal mode is not much separated from the characteristic frequency that corresponds to the $\alpha$-peak maximum in the $G^{\prime \prime}(v)$ curve, clearly demonstrating that no polymer-like elastic modes ${ }^{69}$ exist in addition to the ones that naturally correspond to the $\alpha$-process. Since the time scale of this terminal mode controls the magnitude of viscosity, ${ }^{70}$ it would be worth measuring this quantity independently for such a binary mixture.

While the results of the present solvation study could be explained by considering that only the $\alpha$-relaxation contributes to the polarization fluctuations, a previous investigation on propanol ${ }^{32}$ had been able to identify also the Debye process. Again, the difference between the two cases has its origin in the large spectral separation of Debye-like and primary structural process for the $x=0.5$ mixture. Within the experimental range of the present solvation experiment, only $\tau_{\alpha}(T)$ crosses the time window, $t_{0}$, set by the experiment, whereas $\tau_{\mathrm{D}}(T)$ remains in excess of $2 \mathrm{~s}$ for all temperatures below $150 \mathrm{~K}$. As a result, the Debye-like process does not contribute to the observed redshift and a second solvation step in $v_{\mathrm{e}}(T)$ is expected for $T>150 \mathrm{~K}$, but experimentally inaccessible due to effective oxygen quenching in that range.

A recurring feature in monohydroxy alcohol systems is that nonlinear variations in various properties show up as a function of, e.g., concentration, ${ }^{24,26}$ temperature, ${ }^{25,26}$ pressure, ${ }^{7,68,71}$ and solvation. ${ }^{72}$ This very broad array of observations suggests that the hydrogen-bonded structures which exist in the undiluted bulk phase, at low temperatures, and at low pressures are what one could call "self stabilized," a notion that in studies of hydrogen-bonded systems is well known as cooperative effect. ${ }^{73}$ Now as temperature or pressure are increased, or upon chemical dilution or via surface solvation, the supramolecular structures are destabilized considerably only beyond a certain threshold.

Examples for corresponding effects, reported in the present article, include changes in the concentration dependence of not only $\tau_{D}, \Delta \varepsilon_{D}$, and $\gamma_{D}$ but also in $\tau_{\alpha}$, cf. Fig. 3. These observations hint at what was termed "critical concentration" $x_{c}$, a feature which could also be identified from our NIR measurements on $(2 \mathrm{E} 1 \mathrm{H})_{x}(2 \mathrm{E} 1 \mathrm{Br})_{1-x}$ and $(\mathrm{BuOH})_{x}(\mathrm{BuBr})_{1-x}$ as shown in Fig. 5. A critical concentration, $x_{c} \approx 0.5$, below which the Debye process turns nonexponential, was reported for $2 \mathrm{E} 1 \mathrm{H}$ diluted in bromobutane as well. $^{26}$

It is interesting to compare the concentration dependence of the decoupling ratio for various alcohol-solvent 
mixtures. For $(\mathrm{BuOH})_{x}(\mathrm{BuBr})_{1-x}$ it was found that "the decoupling is smallest for the pure alcohol and increases up to almost a factor of $\tau_{D} / \tau_{\alpha}=10^{4}$ in the dilute limit." ${ }^{24}$ Here, for $(2 \mathrm{E} 1 \mathrm{H})_{x}(2 \mathrm{E} 1 \mathrm{Br})_{1-x}$ the inset of Fig. 3(b) shows that $d(x)$ is maximum for $x \approx 0.5-0.6$ and similar observations were made for $(2 \mathrm{E} 1 \mathrm{H})_{x}(\mathrm{BuBr})_{1-x}$. Also in mixtures of $2 \mathrm{E} 1 \mathrm{H}$ with 3-methylpentane a maximum in $d(x)$ was reported to occur for $x$ close to 0.6 , see Ref. 15. Taken together these results indicate that for $2 \mathrm{E} 1 \mathrm{H}$ the maximum in the decoupling ratio is independent of the solvent, as long as the latter is less viscous than the alcohol, i.e., as long as it has a lower $T_{g}$.

\section{SUMMARY}

In the present article we applied a multitude of experimental techniques, including dielectric, near-infrared, shear mechanical, and solvation spectroscopy in order to study various relaxation phenomena in $(2 \mathrm{E} 1 \mathrm{H})_{x}(2 \mathrm{E} 1 \mathrm{Br})_{1-x}$. When supercooled, these mixtures of branched molecules turned out to be very resistant against crystallization. Therefore, the Debyelike and the structural relaxation could be traced over the entire concentration range using dielectric spectroscopy. We found smooth compositional variations in time scale, dielectric strength, and loss peak broadening as well as an indication of a critical concentration, $x_{c} \approx 0.5-0.6$.

This $x_{c}$ was confirmed by NIR measurements for $(2 \mathrm{E} 1 \mathrm{H})_{x}(2 \mathrm{E} 1 \mathrm{Br})_{1-x}$ and, in harmony with previous dielectric measurements, also for $(\mathrm{BuOH})_{x}(\mathrm{BuBr})_{1-x}$. Furthermore, near $x_{c}$ the Debye-like peak and the structural relaxation of $(2 \mathrm{E} 1 \mathrm{H})_{x}(2 \mathrm{E} 1 \mathrm{Br})_{1-x}$ display an enormous spectral separation of about four decades which facilitates the selective investigation of the various relaxation processes considerably. This selectivity was exploited by studying samples with $x \approx 0.5$ by means of various techniques: The shear mechanical measurements indicate that the Debye process shows no significant viscoelastic signatures and we find clear indications that the rheological response of this hydrogen-bonded system is similar to the one observed for simple liquids. The present solvation experiment, carried out close to the glass transition temperature, was found to monitor the polarization fluctuations corresponding to the $\alpha$-process. Finally, using dielectrically detected physical aging experiments we were able to demonstrate that re-equilibration subsequent to a temperature jump proceeds on the time scale of the structural relaxation and not on that of the Debye-like process.

All in all, the measurements carried out in the present work show that the Debye-like process can be studied profitably not only in neat monohydroxy alcohols but also using suitable diluents in which this process retains most of its characteristic features such as the absence of a detectable rheological signature and the noninvolvement in physical aging. Therefore, the present data conform to approaches recently advanced in order to rationalize the particular features of the Debye process. Moreover, it is not only interesting to study moderately diluted monohydroxy alcohols, as even in the highly diluted limit we find indications for Debye-like features in completely miscible systems such as $(2 \mathrm{E} 1 \mathrm{H})_{x}(2 \mathrm{E} 1 \mathrm{Br})_{1-x}$ or $(\mathrm{BuOH})_{x}(\mathrm{BuBr})_{1-x}$.

\section{ACKNOWLEDGMENTS}

We thank H. J. Weber for his help with the NIR spectrometer. This project was financially supported by the Deutsche Forschungsgemeinschaft under Grant No. BO1301/8 which is highly appreciated. This material is partly based upon the work supported by the National Science Foundation under Grant No. CHE 1026124. The center for viscous liquid dynamics Glass and Time is sponsored by the Danish National Research Foundation (DNRF).

${ }^{1}$ D. Gundermann, U. R. Pedersen, T. Hecksher, N. P. Bailey, B. Jakobsen, T. Christensen, N. B. Olsen, T. B. Schrøder, D. Fragiadakis, R. Casalini, C. M. Roland, J. C. Dyre, and K. Niss, Nat. Phys. 7, 816 (2011).

${ }^{2}$ P. Debye, Verh. Dtsch. Phys. Ges. 15, 777 (1913).

${ }^{3}$ P. Debye, Polar Molecules (The Chemical Catalog Company, New York, 1929), see in particular the work of S. Mizushima as cited in Sec. 19 of the book.

${ }^{4}$ See, e.g., W. Dannhauser and R. H. Cole, J. Chem. Phys. 23, 1762 (1955); S. K. Garg and C. P. Smyth, J. Phys. Chem. 69, 1294 (1965); G. P. Johari and M. Goldstein, J. Chem. Phys. 55, 4245 (1971); C. Hansen, F. Stickel, T. Berger, R. Richert, and E. W. Fischer, ibid. 107, 1086 (1997).

${ }^{5}$ M. A. Floriano and C. A. Angell, J. Chem. Phys. 91, 2537 (1989).

${ }^{6}$ M. W. Sagal, J. Chem. Phys. 36, 2437 (1962); R. Minami, K. Itoh, H. Takahashi, and K. Higasi, ibid. 73, 3396 (1980); C. Brot, Z. Phys. D 11, 249 (1989); U. Kaatze, R. Behrends, and R. Pottel, J. Non-Cryst. Solids 305, 19 (2002).

${ }^{7}$ D. Fragiadakis, C. M. Roland, and R. Casalini, J. Chem. Phys. 132, 144505 (2010)

${ }^{8}$ C. Gainaru, R. Meier, S. Schildmann, C. Lederle, W. Hiller, E. A. Rössler, and R. Böhmer, Phys. Rev. Lett. 105, 258303 (2010).

${ }^{9}$ See, e.g., J. Lehtola, M. Hakala, and K. Hämäläinen, J. Phys. Chem. B 114, 6426 (2010).

${ }^{10}$ P. Sillrén, J. Bielecki, J. Mattsson, L. Börjesson, and A. Matic, J. Chem. Phys. 136, 094514 (2012)

${ }^{11}$ H. Huth, L.-M. Wang, C. Schick, and R. Richert, J. Chem. Phys. 126, 104503 (2007).

${ }^{12}$ S. Schildmann, A. Reiser, R. Gainaru, C. Gainaru, and R. Böhmer, J. Chem. Phys. 135, 174511 (2011).

${ }^{13}$ Substantial progress was achieved some years ago, see Ref. 11.

${ }^{14}$ G. Power, O. E. Panarina, and J. K. Vij, J. Phys.: Condens. Matter 19, 506208 (2007) and papers cited therein.

${ }^{15}$ L. M. Wang, S. Shahriari, and R. Richert, J. Phys. Chem. B 109, 23255 (2005).

${ }^{16}$ G. Power, M. Nagaraj, J. K. Vij, and G. P. Johari, J. Chem. Phys. 134, 044525 (2011).

${ }^{17}$ L. M. Wang and R. Richert, J. Phys. Chem. B 109, 8767 (2005).

${ }^{18}$ A. Schallamach, Trans. Faraday Soc. 42, A180 (1946).

${ }^{19}$ D. J. Denney, J. Chem. Phys. 30, 1019 (1959).

${ }^{20}$ P. Daumezon and R. Heitz, J. Chem. Phys. 55, 5704 (1971).

${ }^{21}$ S. S. N. Murthy and M. Tyagi, J. Sol. Chem. 31, 33 (2002); J. Chem. Phys. 117, 3837 (2002).

${ }^{22}$ G. Power, J. K. Vij, and G. P. Johari, J. Chem. Phys. 127, 094507 (2007).

${ }^{23}$ G. Power, J. K. Vij, and G. P. Johari, J. Chem. Phys. 126, 034512 (2007).

${ }^{24}$ T. El Goresy and R. Böhmer, J. Chem. Phys. 128, 154520 (2008).

${ }^{25}$ C. Lederle, W. Hiller, C. Gainaru, and R. Böhmer, J. Chem. Phys. 134, 064512 (2011).

${ }^{26}$ S. Pawlus, M. Paluch, and M. Dzida, Phys. Rev. E 84, 031503 (2011).

${ }^{27} T_{g}$ for $2 \mathrm{E} 1 \mathrm{Br}$ was estimated from the results of the present work as the temperature at which the dielectric relaxation time is $100 \mathrm{~s}$.

${ }^{28}$ B. Jakobsen, C. Maggi, T. Christensen, and J. C. Dyre, J. Chem. Phys. 129, 184502 (2008)

${ }^{29}$ E. A. DiMarzio and M. Bishop, J. Chem. Phys. 60, 3802 (1974).

${ }^{30}$ K. Niss, B. Jakobsen, and N. B. Olsen, J. Chem. Phys. 123, 234510 (2005); N. Sağlanmak, A. I. Nielsen, N. B. Olsen, J. C. Dyre, and K. Niss, ibid. 132, 024503 (2010).

${ }^{31}$ C. Gainaru, T. Hecksher, N. B. Olsen, R. Böhmer, and J. C. Dyre, J. Chem. Phys. 137, 064508 (2012).

${ }^{32}$ H. Wendt and R. Richert, J. Phys. Chem. A 102, 5775 (1998).

${ }^{33}$ R. Richert, "Local dielectric relaxation by solvation dynamics" in Broadband Dielectric Spectroscopy, edited by F. Kremer and A. Schönhals (Springer, Heidelberg, 2003), pp. 571-594. 
${ }^{34}$ A. Schönhals and F. Kremer, "Analysis of dielectric spectra" in Broadband Dielectric Spectroscopy, edited by F. Kremer and A. Schönhals (Springer, Heidelberg, 2003), p. 71.

${ }^{35}$ G. P. Johari, G. Power, and J. K. Vij, J. Chem. Phys. 117, 1714 (2002).

${ }^{36}$ J. K. Vij and G. Power, J. Non-Cryst. Solids 357, 783 (2011).

${ }^{37}$ C. Gainaru and R. Böhmer, J. Non-Cryst. Solids 356, 542 (2010).

${ }^{38}$ A. Barkatt and C. A. Angell, J. Chem. Phys. 70, 901 (1979).

${ }^{39}$ H. Wagner and R. Richert, J. Phys. Chem. B 103, 4071 (1999).

${ }^{40}$ W. Huang, S. Shahriari, and R. Richert, J. Chem. Phys. 123, 164504 (2005).

${ }^{41}$ T. Christensen and N. B. Olsen, Rev. Sci. Instrum. 66, 5019 (1995).

${ }^{42}$ N. Ito, W. Huang, and R. Richert, J. Phys. Chem. B 110, 4371 (2006); N. Ito and R. Richert, ibid. 111, 5016 (2007).

${ }^{43}$ R. Richert and A. Wagener, J. Phys. Chem. 97, 3146 (1993).

${ }^{44}$ J. G. Kirkwood, J. Chem. Phys. 7, 911 (1939).

${ }^{45}$ S. S. N. Murthy, J. Sobhanadri, and Gangasharan, J. Chem. Phys. 100, 4601 (1994).

${ }^{46}$ F. Qi, R. Böhmer, and H. Sillescu, Phys. Chem. Chem. Phys. 3, 4022 (2001).

${ }^{47}$ J. Workman and L. Weyer, Practical Guide to Interpretive Near-Infrared Spectroscopy (CRC, Boca Raton, 2008), p. 29.

${ }^{48}$ D. H. Rank, H. D. Rix, and T. A. Wiggins, J. Opt. Soc. Am. 43, 157 (1953).

${ }^{49}$ W. Kaye, Spectrochim. Acta 6, 257 (1954).

${ }^{50}$ H. Graener, T. Q. Ye, and A. Laubereau, J. Chem. Phys. 90, 3413 (1989).

${ }^{51}$ M. A. Czarnecki, J. Phys. Chem. A 104, 6356 (2000).

${ }^{52}$ M. Paolantoni, O. Sassi, A. Morresi, and R. S. Cataliotti, Chem. Phys. 310, 169 (2005)

${ }^{53}$ F. Palombo, T. Tassaing, Y. Danten, and M. Besnard, J. Chem. Phys. 125, 094503 (2006)

${ }^{54}$ A. M. C. Davies and S. G. Rutland, Spectrochim. Acta A 44, 1143 (1988).

${ }^{55}$ B. Jakobsen, K. Niss, and N. B. Olsen, J. Chem. Phys 123, 234511 (2005).

${ }^{56}$ C. Maggi, B. Jakobsen, T. Christensen, N. B. Olsen, and J. C. Dyre, J. Phys. Chem. B 112, 16320 (2008).
${ }^{57}$ J. D. Ferry, Viscoelastic Properties of Polymers (Wiley, New York, 1980), Chap. 3, Sec. A1.

${ }^{58}$ H. Vogel, Phys. Z. 22, 645 (1921); G. S. Fulcher, J. Am. Ceram. Soc. 8, 339 (1925); G. Tammann and W. Hesse, Z. Anorg. Allg. Chem. 156, 245 (1926).

${ }^{59}$ The relaxation time may in turn be linked to the mean square displacement, see, e.g., A. Ottochian and D. Leporini, J. Non-Cryst. Solids 357, 298 (2011)

${ }^{60}$ C. Gainaru, S. Kastner, F. Mayr, P. Lunkenheimer, S. Schildmann, H. J. Weber, W. Hiller, A. Loidl, and R. Böhmer, Phys. Rev. Lett. 107, 118304 (2011)

${ }^{61}$ R. Richert and A. Wagener, J. Chem. Phys. 95, 10115 (1991).

${ }^{62}$ F. He and R. Richert, Phys. Rev. B 74, 014201 (2006); W. Wen and R. Richert, J. Chem. Phys. 131, 084710 (2009).

${ }^{63}$ A. Q. Tool, J. Am. Ceram. Soc. 29, 240 (1946); O. S. Narayanaswamy, ibid. 54, 491 (1971); C. T. Moynihan, P. B. Macedo, C. J. Montrose, P. K. Gupta, M. A. DeBolt, J. F. Dill, B. E. Dom, P. W. Drake, A. J. Easteal, P. B. Elterman, R. P. Moeller, H. Sasabe, and J. A. Wilder, Ann. N. Y. Acad. Sci. 279, 15 (1976).

${ }^{64}$ I. M. Hodge, J. Non-Cryst. Solids 169, 211 (1994).

${ }^{65}$ P. Lunkenheimer, R. Wehn, U. Schneider, and A. Loidl, Phys. Rev. Lett. 95, 055702 (2005)

${ }^{66}$ T. Hecksher, N. B. Olsen, K. Niss, and J. C. Dyre, J. Chem. Phys. 133, 174514 (2010).

${ }^{67}$ O. E. Kalinovskaya and J. K. Vij, J. Chem. Phys. 112, 3262 (2000).

${ }^{68}$ S. Pawlus, M. Paluch, M. Nagaraj, and J. K. Vij, J. Chem. Phys. 135, 084507 (2011).

${ }^{69}$ See, e.g., Fig. 10-2 in J. D. Ferry, Viscoelastic Properties of Polymers (Wiley, New York, 1980), Chap. 10, Sec. A1.

${ }^{70}$ G. Harrison, The Dynamic Properties of Supercooled Liquids (Academic, New York, 1976).

${ }^{71}$ A. Reiser, G. Kasper, C. Gainaru, and R. Böhmer, J. Chem. Phys. 132, 181101 (2010).

${ }^{72}$ C. Gainaru, S. Schildmann, and R. Böhmer, J. Chem. Phys. 135, 174510 (2011).

${ }^{73}$ A. Karpfen, Adv. Chem. Phys. 123, 469 (2002). 